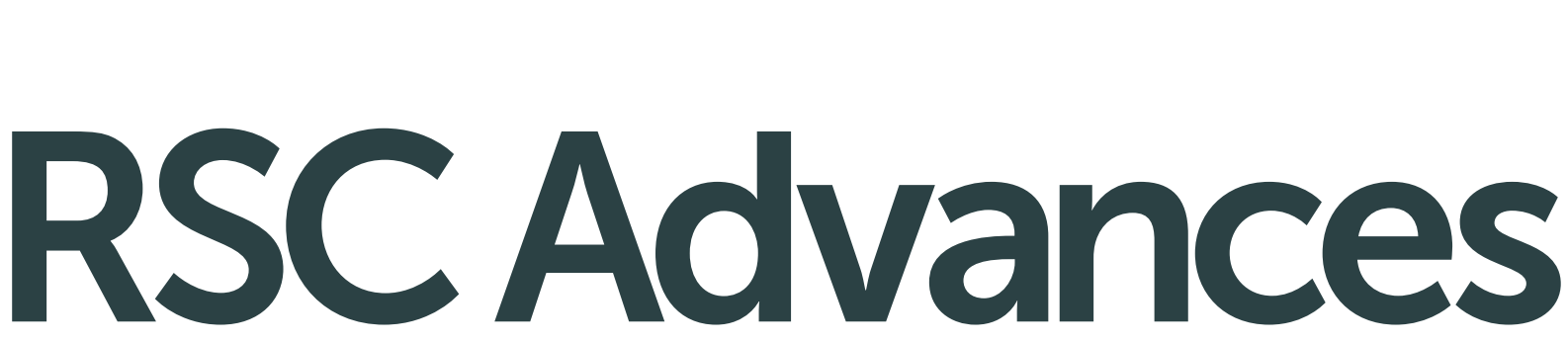

This article can be cited before page numbers have been issued, to do this please use: Z. Yang, S. Chaieb, Y. Hemar, L. deCampo, C. Rehm and D. J. McGillivray, RSC Adv., 2015, DOI:
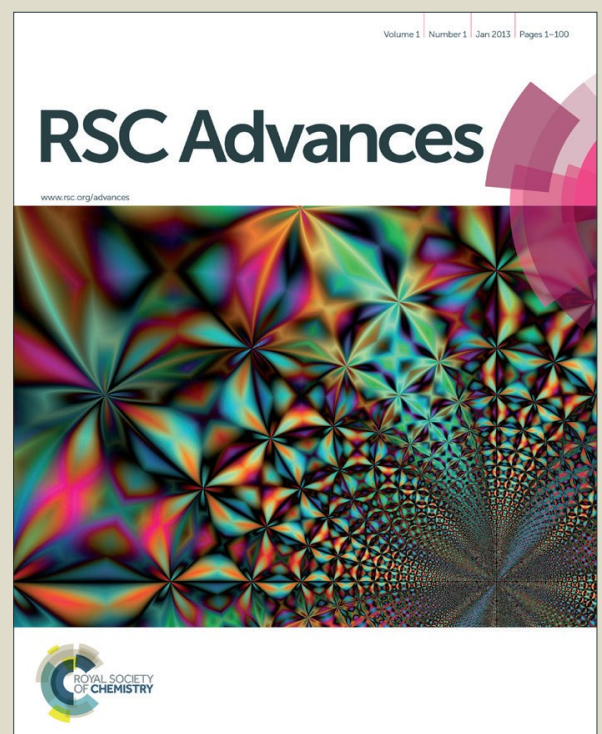

This is an Accepted Manuscript, which has been through the Royal Society of Chemistry peer review process and has been accepted for publication.

Accepted Manuscripts are published online shortly after acceptance, before technical editing, formatting and proof reading. Using this free service, authors can make their results available to the community, in citable form, before we publish the edited article. This Accepted Manuscript will be replaced by the edited, formatted and paginated article as soon as this is available.

You can find more information about Accepted Manuscripts in the Information for Authors.

Please note that technical editing may introduce minor changes to the text and/or graphics, which may alter content. The journal's standard Terms \& Conditions and the Ethical guidelines still apply. In no event shall the Royal Society of Chemistry be held responsible for any errors or omissions in this Accepted Manuscript or any consequences arising from the use of any information it contains. 
30

31

32

33

\section{Investigating Linear and Nonlinear Viscoelastic behaviour and microstructures of Gelatin-Multiwalled carbon nanotubes composites}

Zhi Yang ${ }^{1}$, Sahraoui Chaieb ${ }^{2}$, Yacine Hemar ${ }^{1,3^{*}}$, Liliana de Campo ${ }^{4}$, Christine Rehm $^{4}$ and Duncan J. McGillivray ${ }^{1,5}$

1. School of Chemical Sciences, University of Auckland, Private Bag 92019, Auckland 1142, New Zealand.

2. Division of Physical Sciences and Engineering, King Abdullah University of Science and Technology (KAUST), Thuwal, 23955-6900, Kingdom of Saudi Arabia.

3. The Riddet Institute, Palmerston North, New Zealand

4. Bragg Institute, Australian Nuclear Science and Technology Organisation, Locked Bag 2001, Kirrawee DC, NSW 2232, Australia.

5. MacDiarmid Institute for Advanced Materials and Nanotechnology, PO Box 600, Wellington 6140, New Zealand.

\footnotetext{
*Authors to whom correspondence should be addressed: Email:

y.hemar@auckland.ac.nz
} 


\section{Abstract:}

We have investigated the linear and nonlinear rheology of various gelatin-multiwalled carbon nanotube (gel-MWNT) composites, namely physically-crosslinked-gelatin gel-MWNT composites, chemically-crosslinked-gelatin gel-MWNT composites, and chemicallyphysically-crosslinked-gelatin gel-MWNT composites. Further, the internal structures of these gel-MWNT composites were characterized by ultra-small angle neutron scattering and scanning electron microscopy. The adsorption of gelatin onto the surface of MWNT is also investigated to understand gelatin-assisted dispersion of MWNT during ultrasonication. For all gelatin gels, addition of MWNT increases their complex modulus. The dependence of storage modulus with frequency for gelatin-MWNT composites is similar to that of the corresponding neat gelatin matrix. However, by incorporating MWNT, the dependence of the loss modulus on frequency is reduced. The linear viscoelastic region is decreased approximately linearly with the increase of MWNT concentration. The pre-stress results demonstrate that the addition of MWNT does not change the strain-hardening behaviour of physically-crosslinked gelatin gel. However, the addition of MWNT can increase the strainhardening behaviour of chemically-crosslinked gelatin gel, and chemically-physically crosslinked gelatin gel. Results from light microscopy, cryo-SEM, and USANS demonstrate the hierarchical structures of MWNT, including that tens-of-micron scale MWNT agglomerates are present. Furthermore, the adsorption curve of gelatin onto the surface of MWNT follows two-stage pseudo-saturation behaviour.

Keywords: Gelatin, multiwalled carbon nanotube, composites, Rheology, Ultra-small angle neutron scattering, adsorption 
Introduction:

Carbon nanotubes are long cylinders of covalently-bonded carbon atoms ${ }^{1}$. Since they were applications in the chemical, biochemical, drug controlled release and engineering fields, due to their unique combination of excellent mechanical, electrical, and thermal properties. There are two main types of CNTs available today, namely single walled nanotubes (SWNT) and multi walled nanotubes (MWNT). SWNT can be considered as a single sheet of graphene rolled seamlessly into a cylinder with diameter of order of $1 \mathrm{~nm}$ and length of up to centimetres. MWNT consist of an array of such cylinders formed concentrically and separated by $0.35 \mathrm{~nm}$ with diameter from 2 to $100 \mathrm{~nm}$ and lengths of tens of microns ${ }^{3}$

Carbon nanotubes (CNTs) have been regarded as excellent reinforcing fillers for polymer matrices due to their nanometre size, large aspect ratio (length-to-diameter ratio), extraordinary mechanical strength ${ }^{4}$. This allows a good transfer of load from the matrix to the filler when the composite is put under mechanical stress, in much the same way that steel bars reinforce concrete ${ }^{5}$. Recently, CNTs have been successfully incorporated into various biopolymer hydrogels including hyaluronic acid in the presence of cross-linking reagent divinyl sulfone ${ }^{6}$ or unmodified hyaluronic acid ${ }^{7}$, alginate ${ }^{8}$, chitosan ${ }^{9,10}$, and cyclodextrins 11 .

Gelatin, which forms thermo-reversible gels, is the denatured product of collagen and has been employed as gelling agents and stabilizers in the food and cosmetic industries for a long time ${ }^{12}$. Due to the thermal reversibility of physical gelatin gel, they are not stable at physiological temperature and above, which limits their applications in tissue engineering or other biomedical fields where gels are required to be stable for a certain period of time above room temperature before dissolving. Recently, many studies have investigated chemical or enzymatic cross-linked gelatin gels in order to improve their stability. A variety of crosslinking agents has been employed including transglutaminase ${ }^{13,14}$, glutaraldehyde ${ }^{15}$, phenolic compounds ${ }^{16}$, Bisvinyl sulfonemethyl, genipin ${ }^{17,18}$, and carbodiimides ${ }^{19}$. Here we choose glutaraldehyde as the cross-linker because it is inexpensive, easily available, and has high efficiency for gelatin cross-linking. Other than pure physical and chemical cross linked gelatin gels, several groups have already successfully prepared gelatin gels with the 
102 combination of physical and chemical networks ${ }^{20,21}$. In recent years, gelatin gels have been

103 part of many emerging applications especially in biomedicine area such as encapsulation,

104 tissue scaffold, microspheres, and as matrices for implants ${ }^{22}$. Because it is inexpensive, and

105 has excellent gel forming capability as well as biocompatibility and biodegradability, (cross-

106 linked) gelatin is regarded as one of the most promising candidates for the preparation of

107 CNT-biopolymer composites. In fact, several applications of gelatin-CNTs composites have

108 been reported, and these include the separation of serum proteins ${ }^{23,24}$, haemoglobin

109 immobilization ${ }^{25}$, biosensors for cell detection ${ }^{26}$, (food) packaging material ${ }^{27}$, and cell-

110 laden $3 \mathrm{D}$ constructs ${ }^{28}$.

111

112 It is worth noting that many applications of gelatin-MWNT nanocomposites mainly take 113 advantage of other properties of MWNT (e.g. electrical conductivity ${ }^{29}$, antibacterial activity ${ }^{27}$, 114 cell immobilization ${ }^{30}$, etc...) besides utilizing its well-known reinforcement effect to improve 115 the mechanical properties of gelatin gel. Furthermore, both the processing and application of 116 those gelatin-MWNT nanocomposites require information on their linear and nonlinear 117 rheological properties, which are related to the dispersion state of MWNT, the aspect ratio 118 and orientation of MWNT, the nanocomposites' microstructure, and the interactions between 119 MWNT and polymer chains ${ }^{31}$. Due to the presence of van der Waals attraction between 120 carbon nanotubes together with its hydrophobicity and chemically-smooth surface, CNTs 121 very easily aggregate to form large agglomerates ${ }^{32}$. It is believed that the quality of CNT 122 dispersion, in terms of its stability and the degree of deagglomeration, has a strong impact on 123 the mechanical properties of the final nanocomposites ${ }^{33-35}$. The load transfer between the 124 high-modulus CNT and the polymer matrix depends on the interfacial interaction between the 125 CNT and the matrix. If there is no shear stress or if it acts over distances that are shorter than 126 the length of the CNT or its persistence length, and if there is too much slippage the 127 reinforcement is not optimal and not effective ${ }^{36}$. The properties of this interfacial region 128 depend on the amount of bound polymer to the CNT ${ }^{37}$. The shear stress due to polymer 129 bounding, in the case of a cured urethane/diacrtylate matrix, could be as high as $500 \mathrm{MPa}^{38}$. 130 While CNT had a slight effect on epoxy resins, their effect on compression (23\% increase) 131 was more substantial than on tension where the increase in tensile modulus was under $16 \%{ }^{39}$. 132 This could be due to the buckling of the CNT during compression and their slippage during 133 tension. Rheology provide a unique perspective where the deformation is more complex and 134 sophisticated than a simple tension or compression. Varying the amount of CNT to polymer 
135 as well as varying the nature of the matrix is timely to understand the nature of the 136 reinforcement if any. Also understanding the CNT dispersion and the hierarchical structures 137 of CNT networks in the polymer matrix is extremely important to elucidate the intimate interaction within the composite matrix. To the best of our knowledge, there is no available information on the effect of incorporating MWNT on the mechanical behaviour of various cross-linked and non-cross-linked gelatin gels and their internal structures. Therefore the main aim of this study is to characterize the gelatine-MWNT nanocomposites linear and nonlinear rheological and morphological properties instead of simply improving their mechanical properties.

Materials and Methods:

\section{Materials}

Porcine gelatin powder (bloom value 300, Sigma Aldrich USA), Carboxyl-Multi Walled carbon nanotubes (diameter: 8-15 nm, length: 10-50 $\mu \mathrm{m}$, cheapnanotubes.com, USA), and Glutaraldehyde water solution (Sigma Aldrich USA) were used without further purification.

\section{Methods}

Preparation of Gelatin-MWNT hybrid nanocomposites

The protocols used to prepare the different gelatin-MWNT networks are:

\section{Physically-crosslinked gelatin gel-MWNT composites}

Solutions with a total weight of $5.0 \mathrm{~g}$ containing $2.5 \% \mathrm{w} / \mathrm{w}$ gelatin and one of $0 \% \mathrm{w} / \mathrm{w}, 0.1 \%$ $\mathrm{w} / \mathrm{w}, 0.4 \% \mathrm{w} / \mathrm{w}$ or $1.0 \% \mathrm{w} / \mathrm{w}$ MWNT were prepared using Milli-Q water at $50{ }^{\circ} \mathrm{C}$ under stirring for $1 \mathrm{~h}$, followed by probe sonication (Sonics $750 \mathrm{~W}$, Germany) for 2.5 min using $20 \%$ power amplitude. Samples were then loaded onto a rheometer plate preheated to $50{ }^{\circ} \mathrm{C}$, allowed to equlibrate for $5 \mathrm{~min}$, and then the temperature was decreased from $50{ }^{\circ} \mathrm{C}$ to $20{ }^{\circ} \mathrm{C}$ over 6 minutes $\left(5^{\circ} \mathrm{C} / \mathrm{min}\right)$ to initiate network formation. The physical gelatin-MWNT gel was allowed to form at $20^{\circ} \mathrm{C}$ for $5 \mathrm{~h}$ before conducting rheological measurements.

\section{Chemically-cross-linked gelatin gel-MWNT composites}

165 Chemical networks were formed in the presence of chemical cross-linker glutaraldehyde. The 166 gelatin-MWNT solution was prepared and sonicated in the same conditions as physical gel. 167 After that, we added glutaraldehyde to $2.5 \mathrm{wt} \%$ sonicated gelatin-MWNT solution to achieve $1680.3 \mathrm{wt} \%$ glutaraldehyde vs. total gelatin solution at $35^{\circ} \mathrm{C}$, vortex mixed it at $2000 \mathrm{rpm}$ for 20 
$169 \mathrm{~s}$ (IKA vortex mixer, Germany), and loaded onto the rheometer preheated to $35{ }^{\circ} \mathrm{C}$. This 170 resultant gel cross-linked by glutaraldehyde was left at $35^{\circ} \mathrm{C}$ for $5 \mathrm{~h}$ before conducting rheological measurements.

172

Chemically and-physically crosslinked gelatin-MWNT gel

174

First, chemical networks were made following the above protocol. Subsequently, the temperature of the rheometer plate was cooled from $35{ }^{\circ} \mathrm{C}$ to $20{ }^{\circ} \mathrm{C}$ (at $5{ }^{\circ} \mathrm{C} / \mathrm{min}$ ) to allow for physical networks to form. Samples were left at $20^{\circ} \mathrm{C}$ for an additional $5 \mathrm{~h}$.

Rheology

179

Rheological measurements were carried out on an MCR 302 (Anton Paar GmbH, Graz, Austria) stress-controlled rheometer fitted with a stainless steel plate geometry (diameter: 50 $\mathrm{mm}$ ) set to a gap of $0.50 \mathrm{~mm}$. Sunflower oil was placed around the geometry to minimize water evaporation during measurement. The frequency-sweep measurement was carried out at a constant strain of $1.0 \%$ for frequencies ranging from $10^{-2} \mathrm{~Hz}$ to $10 \mathrm{~Hz}$, and the strainsweep measurement was performed at a constant frequency of $1 \mathrm{~Hz}$ for strains ranging from $10^{-1} \%$ to $10^{4} \%$. In these dynamic measurements the elastic modulus $G^{\prime}$, and the viscous modulus $G^{\prime \prime}$ were obtained.

187

To better quantify the non-linear behaviour of various gelatin-MWNT gels, a differential measurement was utilized. A low amplitude oscillatory stress $\delta \sigma$ was superposed on a constant applied stress $\sigma_{0}$ to determine the differential elastic modulus, $K^{\prime}\left(\sigma_{0}\right)=[\delta \sigma / \delta \gamma]_{\sigma_{0}}$ as a function of $\sigma_{0}$ at $1 \mathrm{~Hz}$. The first applied constant stress (pre-stress) was $1 \mathrm{~Pa}$ in amplitude. Subsequent pre-stresses were, 2, 4, 6, 8, 10, 20, 40, 60, 80, 100, 200, 400, 600, $800,1000,1200,1400,1600,1800,2000,2200$, and $2400 \mathrm{~Pa}$, until the network broke down. At each interval of applied constant stress, small deformation oscillations ( $1 \mathrm{~Pa})$ were conducted at frequencies ranging from $10^{-1} \mathrm{~Hz}$ to $100 \mathrm{~Hz}$ for around 5 minutes. Finally, the differential elastic modulus at $1 \mathrm{~Hz}$ versus the applied constant stress were obtained.

\section{Cryogenic-Scanning electron microscopy (Cryo-SEM)}

199 The microstructure of various gelatin and gelatin-MWNT hydrogels were observed and 200 imaged with a Philips XL30S FEG SEM (Netherlands) using $5 \mathrm{kV}$ accelerating voltage based on the methods of Gaharwar, Dammu et al. 2011 and Molinos, Carvalho et al. 2012. Each 
202 hydrogel sample placed on the stub was plunged into a liquid nitrogen slush $\left(<-196{ }^{\circ} \mathrm{C}\right)$ and 203 then immediately transferred to a Gatan Alto 2500 Cryo Unit (USA) at around $-140{ }^{\circ} \mathrm{C}$. The surface of each frozen sample was fractured using a knife at the same temperature. Fractured samples were etched at $-95{ }^{\circ} \mathrm{C}$ for 30 minutes and then sputter coated with platinum at $120{ }^{\circ} \mathrm{C}$ for $360 \mathrm{~s}$ at $7 \mathrm{~mA}$ (each time $120 \mathrm{~s}$, for three times). Imaging of the fractured surface was completed after placing the etched hydrogel samples on the cryo-stage at $-140{ }^{\circ} \mathrm{C}$.

\section{Optical Microscopy}

Small pieces of various gelatin-MWNT hydrogels were spread onto glass slides to form a thin layer, covered with a coverslip, and then sealed to prevent water evaporation. The microscopic dispersion of MWNT was characterized using an upright Leica DC500 microscope (Germany) in bright field mode with $400 \times$ magnification.

Ultra small angle neutron scattering (USANS)

The sample preparation for the USANS study was the same as that for the rheology study. USANS experiments were performed on the Kookaburra instrument at the OPAL reactor at the Australian Nuclear Science and Technology Organisation (ANSTO), Sydney, Australia ${ }^{40}$. Kookaburra is based on the Bonse-Hart technique ${ }^{41}$ using two sets of identical, 5-bounce, channel-cut, perfect Si single crystals labelled "monochromator" and "analyser" (arranged in long (4.74 $\AA$ ) neutron wavelengths, using 110 and 311 reflection from two channel -cut perfect Si single crystalsxx.

Rocking curve profiles are measured by rotating the analyser crystal away from the aligned peak position (the position in which the undeviated neutrons are reflected into the detector) and measuring the neutron intensity as a function of the momentum transfer, $Q=\frac{4 \pi}{\lambda} \sin \theta$, where $\lambda$ is the wavelength of the incident neutrons and $2 \theta$ is the scattering angle (i.e., the angle of deviation of the scattered neutrons measured from the straight-through beam). $Q$ is measured in a range of $10^{-5}<\mathrm{Q} / \AA^{-1}<10^{-3}$. The USANS data were analysed with SasView (www.sasview.org), accounting for the slit smearing effect by setting the slit height of 0.0584 $\AA^{-1}$. The slopes were determined from original smeared data, then one order of magnitude was subtracted (i.e. $\mathrm{q}^{-2}$ slope become $\mathrm{q}^{-3}$ slope) to account for the slit smearing. 


\section{Gelatin adsorption onto MWNTs}

238 The amount of gelatin absorbed onto the MWNTs was measured using the Bradford method. The MWNTs concentration was set to $0.1 \mathrm{mg} / \mathrm{ml}$ and the gelatin concentration was varied from $0.025 \mathrm{mg} / \mathrm{ml}$ to $0.60 \mathrm{mg} / \mathrm{ml}$. The gelatin-MWNT solutions were probe sonicated (Sonics $750 \mathrm{~W}$, Germany) for 2.5 min using a $20 \%$ power amplitude. After that, the gelatin-MWNT solutions were centrifuged at 10,000 g (SORVALL RC 28S, France) for $1 \mathrm{~h}$ at $35{ }^{\circ} \mathrm{C}$ and the resultant supernatant was used for protein quantification. The centrifuge rotor was preheated to $35{ }^{\circ} \mathrm{C}$ before use. Gelatin solutions of the same concentrations but without MWNTs were sonicated and centrifuged in the same conditions as controls. All supernatants were analysed for protein content using the Bradford assay using a standard calibration curve generated using gelatin. The amount of gelatin absorbed onto the MWNTs was determined by measuring the differences in the concentration of gelatin in the supernatants of gelatin solution alone (control) and gelatin solutions with added MWNTs.

\section{Results and Discussions:}

252

\section{Dynamic rheological behaviour of various gelatin gel-MWNT composites}

The elastic modulus $G^{\prime}$ (solid symbol) and loss modulus $G^{\prime \prime}$ (open symbols) are shown in Fig. 1 as a function of frequency for gelatin physical gel-MWNT composites, gelatin chemical gel-MWNT composites, and gelatin chemical-physical gel-MWNT composites at various MWNT concentrations. These measurements were obtained by applying a constant strain of $1.0 \%$, which is within the linear viscoelastic region. The results demonstrate that for all MWNT concentrations in gelatin-MWTN composites in the applied frequency range, the $G^{\prime}$ is nearly frequency-independent and the $G^{\prime}$ values were greater than the $G^{\prime \prime}$ by at least a factor of 10. This finding suggests that all the gelatin-MWNT samples are gelled with formation of a strong gel network ${ }^{42}$. The viscoelastic behaviour of gelatin-MWNT samples are similar to their corresponding neat gelatin gels, although some differences on the $G^{\prime \prime}$ dependence of frequency can be seen. In all neat gelatin gels, $G^{\prime \prime}$ exhibits a shallow minimum in the low frequency. This behaviour has been seen in various soft solids material including concentrated suspensions, pastes, emulsions, forms, and associative polymers, reflecting the 
269 gelatin gels, the shallow minimum in $G^{\prime \prime}$ disappears. For gelatin physical gel, the high270 frequency power-law dependence of $G^{\prime \prime}$ decreases with increasing MWNT loading, from $f^{0.45}$ for $0.1 \mathrm{wt} \%$ MWNT to $f^{0.27}$ for $1.0 \mathrm{wt} \%$ MWNT. For gelatin chemical and chemicalphysical gels the $G^{\prime \prime}$ is almost independent of frequency at high frequencies when the MWNT loading is higher than $0.4 \mathrm{wt} \%$. This weak dependence of $G^{\prime \prime}$ on frequency suggests the longrange motion and relaxation of gelatin chains are effectively restrained by the presence of $\mathrm{MWNT}^{44}$.

The value of $G^{\prime}$ and $G^{\prime \prime}$ increased with the increase of MWNT loading. To better understand the effect of MWNT loading on the small-deformation rheological behaviour of the gelatinMWNT composites, the complex modulus $G^{*}\left(=\left(\left(G^{\prime}\right)^{2}+\left(G^{\prime}\right)^{2}\right)^{1 / 2}\right.$ at a constant frequency of 1 $\mathrm{Hz}$ as a function of MWNT concentration is reported in Fig. 1 (D). In our studied MWNT concentration range (up to $1 \mathrm{wt} \%$ ), the complex modulus roughly increases linearly with the increase of MWNT concentration for all gelatin matrices, similar to the behaviour observed in poly (propylene fumarate)-SWNT systems ${ }^{45}$. Further we notice that the complex modulus increase by roughly the same amount as a function of the MWNT concentration but the relative increase is the least (30\% instead of $100 \%)$ in the case of the physically cross-linked gels which has an already high complex modulus at $0 \%$ CNT. Strangely enough the critical strain (Fig. 5) decrease more dramatically by more than $55 \%$ in the case of the physically crosslinked gels than for the chemical gels or the hybrid gels where the decrease was modest. Which means that the effect of CNT on the rheological properties of the gel are different than their effect on the structural stability and ultimate strength. These two properties were rather confused in previous works ${ }^{36}$.The viscoelastic behaviour of all gelatin-MWNT composites is still dominated by the gelatin matrix itself. It has been suggested that in dispersions with particle concentrations in excess of percolation $\left(p>>p_{c}\right.$, where $P$ is the volume fraction of the nanoparticles and $p_{c}$ is the value of percolation threshold), the rheology of the composite is dominated by the superstructure of the particles and the modulus of the composites scales as $\left(p-p_{c}\right)^{\delta}$, with $\delta$ ranging between 2.5 and 4.5 for most cases ${ }^{34,46}$. However, as shown in Fig.2, the complex modulus of gelatin-MWNT composites measured here when tested as a function of MWNT concentration cannot be fitted to the power law scaling model which is usually employed for carbon nanotube reinforced polymers ${ }^{47-49}$. This is probably because the highest amount of MWNT used in this study (1 wt \%) did not excess the percolation threshold for gelatin-MWNT composites. To determine the MWNT percolation threshold approximately, we measured the viscosity of MWNT with different concentrations in water after sonication 
303 (Fig. S3). The microstructures of MWNT aggregates were characterized using light

304

305

306

307

308

309

310

311

312

313

314

315

316

317

318

319

320

321

322

323

324

325

326

327

328

329

330

331

332

333

334

335

336 microscopy (Fig. S2). As can be seen in Fig. S3 and S2, when MWNT concentration increased to $1 \mathrm{wt} \%$; the viscosity increased dramatically and MWNT formed fully spanned network. Noted that in this study we focus on the study of addition of MWNT on the small and large deformation rheology of gelatin gel, the overlapping (percolation) of MWNT was deliberately avoided to ensure that the continuous phase is made of gelatin. Therefore, in this study we use $1 \mathrm{wt} \%$ as the highest concentration for MWNT. The reduced reinforcing effect could also be due to aggregation of the MWNT, which would reduce the contact area between filler (MWNT) and matrix (gelatin gel), thus weakening the interfacial stress transfer between them ${ }^{50}$.

\section{Large deformation rheology of various gelatin-MWNT composites}

The strain sweep results performed on gelatin-MWNT samples with various concentration of MWNT are presented in Fig. 2. Qualitatively, for all the gelatin-MWNT samples the behaviour of $G^{\prime}$ and $G^{\prime \prime}$ is similar to the corresponding neat gelatin gel as a function of the applied strain. At low applied strain, within the linear viscoelastic region, $G^{\prime}$ and $G^{\prime \prime}$ were constant with $G^{\prime}$ higher than $G^{\prime \prime}$, suggesting these gelatin-MWNT samples have a solid-like response. When the applied strain is increased further, for all gelatin-MWNT samples $G^{\prime}$ starts to overshoot, depicting a typical strain-hardening behaviour for gelatin gels. At the same time, $G^{\prime \prime}$ increases and reaches a maximum before declining as well. At very high applied strain, both $G^{\prime}$ and $G^{\prime \prime}$ begin to decrease and eventually reach a cross-over point corresponding to the breaking strain. Above that, $G^{\prime \prime}$ is higher than $G^{\prime}$, indicating that flow occurs.

To compare the strain-sweep test on the gelatin-MWNT samples incorporating MWNT, the values of the critical strain and breaking strain are plotted in Fig. 3. For all the samples as the concentration of MWNT is increased the strain amplitude at which nonlinearity begins moves to a lower value. This well-known effect of amplitude dependence of the dynamic viscoelastic properties of filled polymers is often referred as the Payne effect ${ }^{48,51}$. Payne found that the three-dimensional structure network constructed by the aggregation of carbon black significantly altered the dynamic viscoelasticity properties of rubbers ${ }^{51}$. The explanation of this non-linear behaviour is based on two conceptual aspects depending on filler (MWNT) concentration and amplitude deformation. The first mechanism is due to the 
337 filler (MWNT) network breakdown including common features between the 338 phenomenological agglomeration-deagglomeration and recent microscopic networking 339 approaches (particle-particle interaction) as discussed by Heinrich and Kluppel ${ }^{48,52,53}$. The 340 second mechanism is due to polymer chain disentanglements and trapping of polymer chain 341 loops at the filler surface ${ }^{54}$.

342

For physical gelatin-MWNT composites, the breaking (yield) strain amplitudes, above which $G^{\prime}<G^{\prime \prime}$, are around $457 \%, 408 \%, 386 \%$, and $344 \%$ for gelatin-MWNT samples with $0 \mathrm{wt} \%$, $0.1 \mathrm{wt} \%, 0.4 \mathrm{wt} \%$, and $1.0 \mathrm{wt} \%$ MWNT addition respectively. The breaking strain of physical gelatin-MWNT composites is smaller than that of the neat gelatin physical gel, suggesting that the physical gelatin-MWNT composites are somewhat more brittle. Such an embrittlement phenomenon has also been observed in other CNT reinforced polymers like polyimide ${ }^{55}$ and polyetherimide ${ }^{56}$. In contrast with physical gelatin-MWNT composites, for chemical gelatin-MWNT and chemical-physical gelatin-MWNT composites the breaking strain value first increased with the increase of MWNT concentration up to $0.4 \mathrm{wt} \%$ and then decreased. This different break (yielding) behaviour of various gelatin-MWNT composites with MWNT concentrations could be due to the different interfacial interactions between MWNT and gelatin networks and their aggregation and networking within different gelatin gel matrix.

To further characterize the effect of incorporation MWNT on the strain hardening behaviour of various gelatin gels, the Pre-stress protocol was employed. The values of differential elastic modulus $K^{\prime}$ vs. constant applied stress $\sigma$ are shown in Fig. 4. For small values of $\sigma$, the differential elastic modulus is independent of the applied strain and is identical with $G^{\prime}$. As $\sigma$ is increased above some critical value, $\sigma_{c}, K^{\prime}$ increases until the network breaks. In the stress-stiffening regime, we observed that $K^{\prime} \sim \sigma^{1.1}$ for gelatin physical gel alone and gelatin physical gel-MWNT composites, as shown in Fig. 4A. The incorporation of MWNT into gelatin physical gel does not change its strain hardening behaviour. For gelatin chemical gel, the incorporation of MWNT changed the power scaling exponent from 0.65 for gelatin chemical gel alone to around 0.84 once MWNT is incorporated, as shown in Fig. 4B. This result suggests that incorporation of MWNT increases the strain hardening for gelatin chemical gel. For chemical-physical gel alone, $K^{\prime}$ is expressed with two power laws. In the 369 lower stress region, $K^{\prime} \sim \sigma^{0.65}$; while in the higher stress region, $K^{\prime} \sim \sigma^{1.5}$. The incorporation 
of MWNT changed the power scaling exponent in the low stress region from 0.65 for the gelatin chemical-physical gel alone to around $0.70,0.84$, and 0.84 for MWNT concentration $0.1 \mathrm{wt} \%, 0.4 \mathrm{wt} \%$, and $1.0 \mathrm{wt} \%$, respectively. This result indicates that the incorporation of MWNT enhanced the strain hardening for gelatin chemical-physical gel. At the very highest stresses, for chemical gelatin gel-MWNT and chemical-physical gelatin gel-MWNT with MWNT concentration $0.4 \mathrm{wt} \%$, and $1.0 \mathrm{wt} \%$; the experimental data deviates from the power law scaling as indicated with solid line in Fig. 4. This deviation could result from irreversible network fracture or failure ${ }^{57}$. To compare the strain sweep measurement and pre-stress measurements of the various gelatin-MWNT composites with different added MWNT, the critical stress values obtained from these two measurements are shown in Fig. 5. The critical stress values obtained from the strain sweep and pre-stress agree well and decrease linearly with the increase of MWNT content, suggesting again that with increasing MWNT loading, the polymer nanocomposites get stiffer and more fragile. Such behaviour is typical of fractal networks such as those of colloidal gels, layered silicates, and flocculated silica spheres ${ }^{58}$.

\section{Highly Porous Gelatin-MWNT composites networks revealed by Cryo-SEM}

Structural information about gelatin-MWNT composites, such as the extent of MWNT aggregation and phase separation, is extremely important for understanding their rheological properties and in formulating the composites to meet further application requirements. CryoSEM has been used extensively to characterize hydrogel and hydrogel nanocomposite structures. For example, the porous structures of hydrated gelatin and agar gels ${ }^{59}$ and incorporation of dextrin nanoparticles into dextrin hydrogel can be visualized using cryoSEM ${ }^{60}$. However, it is worth noting that cryo-SEM does not image the true wet hydrogel architecture itself but instead the collapsed hydrogel structure after etching (where etching involves semi drying) ${ }^{61}$. Despite these limitations, this technique still gives rough structural information related to the original hydrogel state, and especially of the extent of MWNT aggregation within various gelatin matrices.

The various gelatin gel-MWNT composites were examined by cryo-SEM and typical results are demonstrated in Fig. 6. Darker areas in the images correspond to amorphous water which was not sublimated during the sample preparation process, while lighter objects correspond to gelatin structures after etching ${ }^{62}$. All of the nanocomposites have an interconnected porous structure with pore sizes in the range of about 1-8 $\mu \mathrm{m}$. For gelatin physical gel, incorporation 
404

405

406

407

408

409

410

411

412

413

414

415

416

417

418

419

420

421

422

423

424

425

426

427

428

429

430

431

432

433

434

435

436

of $0.4 \mathrm{wt} \%$ MWNT increases the pore size from about $2 \mu \mathrm{m}$ to $5 \mu \mathrm{m}$. This may be due to a reduction in the amount of gelatin available for gelation after adsorption onto the surface of the MWNT. It is also possible that the incorporation of MWNT reduces the gelatin diffusion. Both effects would reduce the gel nucleation rate, which has been suggested to produce larger pores $^{63}$. The increase of pore size with addition of MWNT is also observed in chemical gel, however it is not obvious in chemical-physical gel.

The SEM images from the various gelatin gel having 0.4 and $1.0 \mathrm{wt} \%$ MWNT concentrations demonstrate the presence of strong structural heterogeneity, which may be induced by the aggregation of MWNT $(5-30 \mu \mathrm{m})$ or water evaporation during sample preparation ${ }^{61}$. There are more and larger MWNT aggregates present in gelatin gels with higher (1.0wt\%) MWNT concentration. The presence of such micron-scale MWNT agglomerates is also confirmed by optical microscope images (Fig. 7).

\section{Ultra-small angle neutron scattering (USANS)}

Although various microscopic techniques including atomic force microscopy (AFM) ${ }^{64}$, optical bright-field and dark-field optical microscopy ${ }^{34}$, scanning and transmission electron microscopy (SEM and TEM) ${ }^{65}$ have been employed to visualize the CNTs and their agglomerates, the various sample preparations by drop-casting, etching, or freeze drying may have significant influences on the arrangement of CNTs and cause structural artefacts ${ }^{32}$. Therefore, we have employed USANS to further study the hierarchical structures of MWNT network in the composites in situ.

USANS is a probe that allows the characterization of micron-scale structures up to several tens of microns ${ }^{33}$. Recently, USANS has been employed to characterize the hierarchical structures of carbon nanotubes networks and their dispersion in various polymer and ceramic matrices $33-35,66$. Our USANS data (Fig. 8) have been obtained using both short and long neutron wavelengths $\left(2.37 \AA\right.$ and $4.74 \AA$ respectively) in the range of $1.8 \times 10^{-5}<\mathrm{Q} / \AA^{-1}<$ $0.01 \AA^{-1}$, corresponding to a probed length scale of $60 \mathrm{~nm}$ up to about $35 \mu \mathrm{m}$. It is worth noting that the neutron contrast between $\mathrm{H}_{2} \mathrm{O}$ and the gelatin in the gelatin-MWNT composites is very low, such that the majority of the scattering arises from the MWNT networks only rather than aqueous voids ${ }^{67}$. 
438 The scattering intensities of MWNT aggregates exhibit a scattering intensity, $I$ (q), that 439 follows a power law equation given as.

$$
I(q)=\frac{A}{q^{m}}+B
$$

440 Where from $m$, the power exponent, the nature of the scattering object can be deduced ${ }^{68}$. For 441 example, $m=1$ indicates thin rods or filaments, $m=2$ indicates thin platelet and $2<m<3$ 442 may refer to mass fractal structures (three dimensional self-similarity over a large range of 443 length scales), and $3 \leq m<4$ corresponds to surface fractal structures (rough surfaces with 444 self-similarity over a large range of length scales) ${ }^{69}$. Across the USANS $q$-range, both 445 gelatin-MWNT gels demonstrate three power law dependences $(\alpha, \beta$, and $\gamma)$, and are identical 446 except at the lowest q-range (corresponding to the largest length scales). For $q$ range from $4471.8 \times 10^{-5}$ to $1.0 \times 10^{-3} \AA^{-1}$, probing length scales $>5 \mu \mathrm{m}$, the power law exponent for gelatin 448 physical gel-MWNT composites $\left(\alpha_{1}\right)$ and gelatin chemical-physical gel-MWNT composites $449\left(\alpha_{2}\right)$ is 2.6 and 2.2 respectively. Thus both gelatin-MWNT composites exhibit a mass fractal 450 behaviour at the largest length scales due to the presence of disordered networks of bulk 451 MWNT aggregates ${ }^{33,35}$. The higher value for the exponent for the gelatin physical gel452 MWNT indicates a denser network than for the chemical-physical gel composites ${ }^{70}$. In the $q$ 453 range of $1.0 \times 10^{-4}$ to $1.0 \times 10^{-3} \AA^{-1}$, an identical power law exponent of $\beta=3.2 \mathrm{can}$ be observed 454 for both gelatin chemical-physical gel-MWNT composites and gelatin physical gel-MWNT 455 composites. This scattering can be interpreted as surface fractal behaviour at probe lengths of $456 \sim 0.5 \mu \mathrm{m}$ to $5 \mu \mathrm{m}$. In this case, $D s=6-\beta$, where $D s$ is the surface fractal dimension, which 457 ranges from 2 for a smooth surface to 3 for a uniformly dense object that is entirely surface 458 (something like crumpled paper) ${ }^{71}$. The observed power law regime with $\beta=3.2$, or $D s=2.8$, 459 demonstrates that the MWNT aggregates have a high surface area to volume ratio. At higher $460 q$-values, from $1.0 \times 10^{-3}$ to $1.0 \times 10^{-2} \AA^{-1}\left(<0.5 \mu \mathrm{m}\right.$ probe length) there is a $q^{-1.7}$ dependence 461 for both gelatin gel-MWNT composites, due to the presence of a disordered but loose 462 network of MWNT ${ }^{33}$.

463

464 There is no evidence of structure factor scattering in the USANS data, which confirms that 465 the dispersion of the MWNT is random, with no characteristic spacing between clusters in 466 this length range. It is also worth noting that there is no region in the measured USANS 467 profile exhibiting power-law scattering with an exponent of -1 , which is characteristic of a 468 dispersion of long rod-like particles. A well-dispersed and unaggregated dispersion of 
469 MWNT would contain a wide region in which $I(q)$ scales in proportion with $q^{-1}$, however,

470

471

472

473

474

475

476

477

478

479

480

481

482

483

484

485

486

487

488

489

490

491

492

493

494

495

496

497

498

499

500

501

502 such a perfect dispersion is only found rarely under dilute conditions, with a large quantity of dispersant ${ }^{70,72}$. In most studies of the dispersion of CNT by (U)SAXS and (U)SANS, powerlaw scattering with an exponent of -1 is absent, and dense fractal networks and/or surface fractal characteristics are found instead ${ }^{32-35}$. In this, our USANS results agree with previous studies in demonstrating poor dispersion of the MWNTs within the gelatin matrix, and the presence of micron scale fractal (mass and surface) structures within the composites. This confirms the cryo-SEM results and helps to explain the poor reinforcement of the mechanical properties revealed by the rheological studies.

\section{Absorption of gelatin on the surface of MWNTs}

Investigating the adsorption of polymers (proteins, DNA, and polysaccharides) onto the surface of MWNTs is important both in the development of nanoscale biosensors and biocatalytic devices ${ }^{73}$ and in understanding polymer-assisted dispersion of carbon nanotubes 74. The adsorption of gelatin onto MWNT as a function of the amount of the gelatin is presented in Fig. 9. The adsorption of gelatin follows a two stage pseudo-saturation behaviour, with the amount of gelatin attached to the MWNT increasing with gelatin concentration until a plateau value of around $0.8 \mathrm{mg}$ gelatin $/ \mathrm{mg}$ MWNT is reached at a gelatin concentration of $\sim 0.3 \mathrm{mg} / \mathrm{ml}$. When the gelatin concentration is increased beyond a critical value at $\sim 0.4 \mathrm{mg} / \mathrm{ml}$, the amount of adsorbed gelatin on MWNT increased rapidly again until a second plateau of $1.4 \mathrm{mg}$ gelatin $/ \mathrm{mg}$ MWNT is reached at $\sim 0.5 \mathrm{mg} / \mathrm{ml}$ gelatin concentration. The one stage pseudo-saturation adsorption behaviour has also been observed with other proteins attaching to carbon nanotubes including soybean peroxidase ${ }^{75}$ and bovine serum albumin ${ }^{76}$. The appearance of the second pseudo-saturation region could be due to the fact that during sonication when the exposed gelatin concentration increases; the large bundles or agglomerates of MWNTs can be disintegrate into small bundles and individual tubes (as revealed by USANS), thus increasing the area of MWNTs for more gelatin adsorption.

It is believed that the driving force for protein adsorption on carbon nanotubes is mainly due to both hydrophobic interactions and the ability to form $\pi-\pi$ stacking interactions between 
503 aromatic residues and the carbon nanotubes ${ }^{77-79}$. Given the lack of aromatic amino acids in

504 gelatin molecules, the adsorption of gelatin on MWNTs must be mainly due to hydrophobic

505 interactions and the interaction between $\mathrm{COOH}$ (functionalized group on the surface of

506 MWNTs) and amino acid within gelatin.

507

\section{Conclusions}

509 From the above results into the rheology and structure of the MWNT and gelatin complexes,

510 several points are clear: 1) by incorporating MWNT into gelatin matrices at loadings of up to

$5111 \mathrm{wt} \%$, the complex modulus (at $1 \mathrm{~Hz}$ ) of the composite is weakly increased proportional to

512 the loading; 2) The $G^{\prime}$ dependence on frequency of all of these gelatin-MWNT composites is

513 still dominated by the corresponding gelatin matrix. However, the loss modulus $G^{\prime \prime}$ becomes

514 less frequency dependent when MWNT is incorporated into the gelatin matrix, suggesting

515 that the long-range motions and relaxations of the gelatin chains are effectively restrained by

516 the presence of the MWNT; 3) The value of the critical strain (stress), at which the linear

517 viscoelastic region ends, decreases roughly linearly with increasing MWNT loading; 4) The

518 pre-stress study demonstrates that for the physically-crosslinked gelatin gels, the addition of

519 MWNT does not change their strain hardening behaviour. However, for chemically-

520 crosslinked and chemically-physically crosslinked gelatin gels the addition of MWNT

521 increases their strain hardening behaviour; 5) The USANS result showed that there are three

522 levels of hierarchical structures of MWNT networks within physically-crosslinked gelatin gel

523 and chemically and physically-crosslinked gelatin gels. Tens-of-micron scale randomly

524 distributed MWNT agglomerates are present, confirming the poor dispersion and large

525 aggregation of MWNT in the various gelatin matrices seen by Cryo-SEM and light

526 microscopy study; and finally, 6) The adsorption of gelatin onto surface of MWNT during

527 ultrasonication demonstrates two regions of pseudo-saturation behaviour.

529 Overall, it is clear that the MWNT are not fully dispersed in the gelatin gels, but still

530 influence the linear and nonlinear mechanical behaviour of various gelatin gels, and even the

531 pore size distribution and structure. It is also clear that the gelatin is interacting strongly with

532 the MWNT, as the significant gelatin loadings on the tubes shows, and that chemical gelation

533 increases the impact of the MWNT on the interaction between the gel and the MWNT. 


\section{Acknowledgements}

540 The authors acknowledge travel support for these experiments from the Australian Institute of with Cryo-SEM and Adrian Turner for assistance with light microscopy. SC and ZY thanks KAUST for financial support.

\section{References}

548 1. M. Moniruzzaman and K. I. Winey, Macromolecules, 2006, 39, 5194-5205.

2. S. Iijima, nature, 1991, 354, 56-58.

550 3. J. N. Coleman, U. Khan, W. J. Blau and Y. K. Gun'ko, Carbon, 2006, 44, 1624-1652.

4. H. Wang, W. Zhou, D. L. Ho, K. I. Winey, J. E. Fischer, C. J. Glinka and E. K. Hobbie, Nano Letters, 2004, 4, 1789-1793.

5. P. M. Ajayan and J. M. Tour, Nature, 2007, 447, 1066-1068.

6. S. Bhattacharyya, S. Guillot, H. Dabboue, J.-F. Tranchant and J.-P. Salvetat, Biomacromolecules, 2008, 9, 505-509.

7. C. Zamora-Ledezma, L. Buisson, S. E. Moulton, G. Wallace, C. Zakri, C. Blanc, E. Anglaret and P. Poulin, Langmuir, 2013, 29, 10247-10253.

8. Z. Jiang, S. Xu, Y. Lu, W. Yuan, H. Wu and C. Lv, Journal of Biomaterials Science, Polymer Edition, 2006, 17, 21-35.

9. S. Chatterjee, M. W. Lee and S. H. Woo, Bioresource technology, 2010, 101, 1800-1806.

10. S.-F. Wang, L. Shen, W.-D. Zhang and Y.-J. Tong, Biomacromolecules, 2005, 6, 3067-3072.

11. T. Ogoshi, Y. Takashima, H. Yamaguchi and A. Harada, Journal of the American Chemical Society, 2007, 129, 4878-4879.

12. F. Bode, M. A. da Silva, A. F. Drake, S. B. Ross-Murphy and C. A. Dreiss, Biomacromolecules, 2011, 12, 3741-3752.

13. H. Babin and E. Dickinson, Food Hydrocolloids, 2001, 15, 271-276.

14. N. F. Mohtar, C. O. Perera, S.-Y. Quek and Y. Hemar, Food Hydrocolloids, 2013, 31, 204209.

15. B.-S. Chiou, R. J. Avena-Bustillos, J. Shey, E. Yee, P. J. Bechtel, S. H. Imam, G. M. Glenn and W. J. Orts, Polymer, 2006, 47, 6379-6386. 
571 16. X. Zhang, M. D. Do, P. Casey, A. Sulistio, G. G. Qiao, L. Lundin, P. Lillford and S. Kosaraju,

573 17. D. M. Kirchmajer, C. A. Watson and M. Ranson, RSC Advances, 2013, 3, 1073-1081.

18. A. Bigi, G. Cojazzi, S. Panzavolta, N. Roveri and K. Rubini, Biomaterials, 2002, 23, $4827-$ 4832 .

20. F. Bode, M. A. da Silva, P. Smith, C. D. Lorenz, S. McCullen, M. M. Stevens and C. A. Dreiss, soft matter, 2013.

22. L. Hough, M. Islam, B. Hammouda, A. Yodh and P. Heiney, Nano letters, 2006, 6, 313-317.

23. H. Li, D. Wang, B. Liu and L. Gao, Colloids and Surfaces B: Biointerfaces, 2004, 33, 85-88.

24. H. Li, D. Q. Wang, H. L. Chen, B. L. Liu and L. Z. Gao, Macromolecular Bioscience, 2003, 3, 720-724.

25. W. Zheng and Y. Zheng, Electrochemistry communications, 2007, 9, 1619-1623.

26. J.-J. Zhang, M.-M. Gu, T.-T. Zheng and J.-J. Zhu, Analytical chemistry, 2009, 81, 6641-6648.

27. M. Ortiz-Zarama, A. Jiménez-Aparicio, M. Perea-Flores and J. Solorza-Feria, Journal of Food Engineering, 2014, 120, 223-232.

31. D. Wu, L. Wu, Y. Sun and M. Zhang, Journal of Polymer Science Part B: Polymer Physics, 2007, 45, 3137-3147.

32. A. A. Golosova, J. Adelsberger, A. Sepe, M. A. Niedermeier, P. Lindner, S. S. Funari, R. Jordan and C. M. Papadakis, The Journal of Physical Chemistry C, 2012, 116, 15765-15774.

33. O. Tapasztó, H. Lemmel, M. Markó, K. Balázsi, C. Balázsi and L. Tapasztó, Chemical Physics Letters, 2014, 614, 148-150.

34. T. Chatterjee, A. Jackson and R. Krishnamoorti, Journal of the American Chemical Society, 2008, 130, 6934-6935.

35. D. W. Schaefer and R. S. Justice, Macromolecules, 2007, 40, 8501-8517.

36. P. M. Ajayan, L. S. Schadler, C. Giannaris and A. Rubio, Advanced Materials, 2000, 12, 750753.

604 37. G. Tsagaropoulos and A. Eisenberg, Macromolecules, 1995, 28, 6067-6077. 
605 38. H. Wagner, O. Lourie, Y. Feldman and R. Tenne, Applied physics letters, 1998, 72, 188-190.

606 39. L. Schadler, S. Giannaris and P. Ajayan, Applied physics letters, 1998, 73, 3842-3844.

607

608

609

610

611

612

613

614

615

616

617

618

619

620

621

622

623

624

625

626

627

628

629

630

631

632

633

634

635

636

637

40. C. Rehm, A. Brûlé, A. K. Freund and S. J. Kennedy, Journal of Applied Crystallography, 2013, 46, 1699-1704.

41. U. Bonse and M. Hart, Applied Physics Letters, 1965, 7, 238-240.

42. S. P. R. Lapasin, Rheology of industrial polysaccharides: Theory and applications, 1995.

43. H. M. Wyss, K. Miyazaki, J. Mattsson, Z. Hu, D. R. Reichman and D. A. Weitz, Physical review letters, 2007, 98, 238303.

44. F. Du, R. C. Scogna, W. Zhou, S. Brand, J. E. Fischer and K. I. Winey, Macromolecules, 2004, 37, 9048-9055.

45. X. Shi, J. L. Hudson, P. P. Spicer, J. M. Tour, R. Krishnamoorti and A. G. Mikos, Nanotechnology, 2005, 16, S531.

46. V. Trappe and D. Weitz, Physical review letters, 2000, 85, 449.

47. Z. Zhu, T. Thompson, S.-Q. Wang, E. D. von Meerwall and A. Halasa, Macromolecules, $2005, \mathbf{3 8}, 8816-8824$.

48. P. Cassagnau, Polymer, 2008, 49, 2183-2196.

49. E. K. Hobbie, Rheologica acta, 2010, 49, 323-334.

50. T. Domenech, R. Zouari, B. Vergnes and E. Peuvrel-Disdier, Macromolecules, 2014, 47, 3417-3427.

51. Payne, Reinforcement of elastomers., New York: Interscience, 1965.

52. G. Heinrich and M. Klüppel, in Filled elastomers drug delivery systems, Springer, 2002, pp. $1-44$.

53. P. Cassagnau, Polymer, 2003, 44, 2455-2462.

54. S. Sternstein and A.-J. Zhu, Macromolecules, 2002, 35, 7262-7273.

55. J. J. Ge, D. Zhang, Q. Li, H. Hou, M. J. Graham, L. Dai, F. W. Harris and S. Z. Cheng, Journal of the American Chemical Society, 2005, 127, 9984-9985.

56. Y. Chen, J. Tao, L. Deng, L. Li, J. Li, Y. Yang and N. M. Khashab, ACS applied materials \& interfaces, 2013, 5, 7478-7484.

57. Y.-C. Lin, C. P. Broedersz, A. C. Rowat, T. Wedig, H. Herrmann, F. C. MacKintosh and D. A. Weitz, Journal of Molecular Biology, 2010, 399, 637-644.

58. T. Chatterjee and R. Krishnamoorti, Soft Matter, 2013, 9, 9515-9529.

59. J. Rahbani, A. R. Behzad, N. M. Khashab and M. Al - Ghoul, Electrophoresis, 2013, 34, 405408. 
60. M. Molinos, V. Carvalho, D. M. Silva and F. M. Gama, Biomacromolecules, 2012, 13, $517-$ 527.

61. A. K. Gaharwar, S. A. Dammu, J. M. Canter, C.-J. Wu and G. Schmidt, Biomacromolecules, 2011, 12, 1641-1650.

62. L. Hilliou, M. Wilhelm, M. Yamanoi and M. P. Gonçalves, Food Hydrocolloids, 2009, 23, 2322-2330.

63. S. Van Vlierberghe, V. Cnudde, P. Dubruel, B. Masschaele, A. Cosijns, I. De Paepe, P. J. S. Jacobs, L. Van Hoorebeke, J. P. Remon and E. Schacht, Biomacromolecules, 2007, 8, 331337.

64. M.-F. Yu, O. Lourie, M. J. Dyer, K. Moloni, T. F. Kelly and R. S. Ruoff, Science, 2000, 287, 637-640.

65. P. Pötschke, T. D. Fornes and D. R. Paul, Polymer, 2002, 43, 3247-3255.

66. Y. Dror, W. Pyckhout-Hintzen and Y. Cohen, Macromolecules, 2005, 38, 7828-7836.

67. M. Helminger, B. Wu, T. Kollmann, D. Benke, D. Schwahn, V. Pipich, D. Faivre, D. Zahn and H. Cölfen, Advanced Functional Materials, 2014, 24, 3187-3196.

68. P. W. Schmidt, Journal of Applied Crystallography, 1991, 24, 414-435.

69. F. Xia, J. Zhao, B. E. Etschmann, J. Brugger, C. J. Garvey, C. Rehm, H. Lemmel, J. Ilavsky, Y.-S. Han and A. Pring, American Mineralogist, 2014, 99, 2398-2404.

70. M. J. Hollamby, Physical Chemistry Chemical Physics, 2013, 15, 10566-10579.

71. D. W. Schaefer, J. Zhao, J. M. Brown, D. P. Anderson and D. W. Tomlin, Chemical Physics Letters, 2003, 375, 369-375.

72. S. Fogden, C. A. Howard, R. K. Heenan, N. T. Skipper and M. S. P. Shaffer, ACS Nano, 2012, 6, 54-62.

73. Y. Lin, L. F. Allard and Y.-P. Sun, The Journal of Physical Chemistry B, 2004, 108, 3760 3764 .

74. S. S. Karajanagi, H. Yang, P. Asuri, E. Sellitto, J. S. Dordick and R. S. Kane, Langmuir, 2006, 22, 1392-1395.

75. S. S. Karajanagi, A. A. Vertegel, R. S. Kane and J. S. Dordick, Langmuir, 2004, 20, $11594-$ 11599.

76. L. E. Valenti, P. A. Fiorito, C. D. García and C. E. Giacomelli, Journal of colloid and interface science, 2007, 307, 349-356.

77. C. Ge, J. Du, L. Zhao, L. Wang, Y. Liu, D. Li, Y. Yang, R. Zhou, Y. Zhao and Z. Chai, Proceedings of the National Academy of Sciences, 2011, 108, 16968-16973.

78. K. Matsuura, T. Saito, T. Okazaki, S. Ohshima, M. Yumura and S. Iijima, Chemical Physics Letters, 2006, 429, 497-502.

79. S. A. Bhakta, E. Evans, T. E. Benavidez and C. D. Garcia, Analytica Chimica Acta, 2015, 872, $7-25$. 


\section{Figure captions:}

Fig.1. Elastic modulus $G^{\prime}$ (solid symbol) and loss modulus $G^{\prime \prime}$ (open symbols) as a function of frequency for (A) gelatin physical gel-MWNT composites (measured at $20^{\circ} \mathrm{C}$ ), (B) gelatin chemical gel-MWNT composites (measured at $35^{\circ} \mathrm{C}$ ), and (C) gelatin chemical-physical gelMWNT composites (measured at $20^{\circ} \mathrm{C}$ ). MWNT concentrations are: $0 \%(\boldsymbol{\bullet}, \square) ; 0.1 \%(\bullet, \circ)$; $0.4 \%(\Delta, \triangle)$; and $1.0 \%(\nabla, \nabla)$. (D) The complex modulus $G^{*}$ at $1 \mathrm{~Hz}$ as a function of MWNT concentration for gelatin physical gel-MWNT composites (घ), gelatin chemical gelMWNT composites $(\bullet)$, and (C) gelatin chemical-physical gel-MWNT composites $(\triangleleft)$.

Fig.2. Elastic modulus $G^{\prime}$ (solid symbol) and loss modulus $G^{\prime \prime}$ (open symbols) as a function of strain (\%) for (A) gelatin physical gel-MWNT composites (measured at $20^{\circ} \mathrm{C}$ ), (B) gelatin chemical gel-MWNT composites (measured at $35^{\circ} \mathrm{C}$ ), and $(\mathrm{C})$ gelatin chemical-physical gelMWNT composites (measured at $20^{\circ} \mathrm{C}$ ). MWNT concentrations are: $0 \%(\boldsymbol{\bullet}, \square) ; 0.1 \%(\bullet, \circ)$; $0.4 \%(\Delta, \triangle)$; and $1.0 \%(\nabla, \nabla)$.

Fig.3. The critical strain $\left(\gamma_{\text {linear }}\right)$ values (solid symbols) and breaking strain values (empty symbols) as a function of concentration of MWNT for (A) gelatin physical gel-MWNT composites (measured at $20^{\circ} \mathrm{C}$ ), (B) gelatin chemical gel-MWNT comoposites (measured at $35^{\circ} \mathrm{C}$ ), and (C) gelatin chemical-physical gel-MWNT composites (measured at $20^{\circ} \mathrm{C}$ ).

Fig.4. The differential elastic modulus $K^{\prime}$, as a function of applied constant shear stress, $\sigma_{0}$ for (A) gelatin physical gel-MWNT composites (measured at $20^{\circ} \mathrm{C}$ ), (B) gelatin chemical gel-MWNT composites (measured at $35^{\circ} \mathrm{C}$ ), and (C) gelatin chemical-physical gel-MWNT composites (measured at $20^{\circ} \mathrm{C}$ ). MWNT concentrations are: $0 \%(\mathbf{\square}, \square) ; 0.1 \%(\bullet, \circ) ; 0.4 \%$ $(\Delta, \Delta)$; and $1.0 \%(\nabla, \nabla)$. The solid line and number indicates the power law scaling of $K^{\prime}$ vS. $\sigma_{0}$.

Fig.5. Critical stress values of gelatin physical gel-MWNT composites ( $\mathbf{\square}, \square)$, gelatin chemical gel-MWNT composites $(\bullet, \circ)$, and gelatin chemical-physical gelatin gel-MWNT composites $(\triangleleft, \triangleleft)$ as function of MWNT concentration obtained from pre-stress (solid symbol) and strain (stress) sweep (empty symbol). 


\section{Fig. 6}

Cryo-SEM images from (A) physical gelatin gel, (B) chemical gelatin gel, and (C) chemicalphysical gelatin gel with different concentration of MWNT incorporation. The MWNT aggregates are indicated by arrows.

Fig. 7

Optical microscope image of various gelatin gels with incorporation of $0.4 \mathrm{wt} \%$ and $1.0 \mathrm{wt} \%$ of MWNT. Scale bars represent 100 micron.

\section{Fig. 8}

Ultra-small angle neutron scattering (USANS) scattering intensities as a function of scattering wavenumber for physically-crosslinked gelatin gel-MWNT composites (black symbol), and chemically- physically crosslinked gelatin gel-MWNT composites (red symbol). The solid symbol describe the USANS pattern obtained at a wavelength of $2.37 \AA$, while the empty symbol is from a wavelength of $4.74 \AA$. The solid lines indicate the power law fitting regions of the data. 


\section{Fig. 1.}
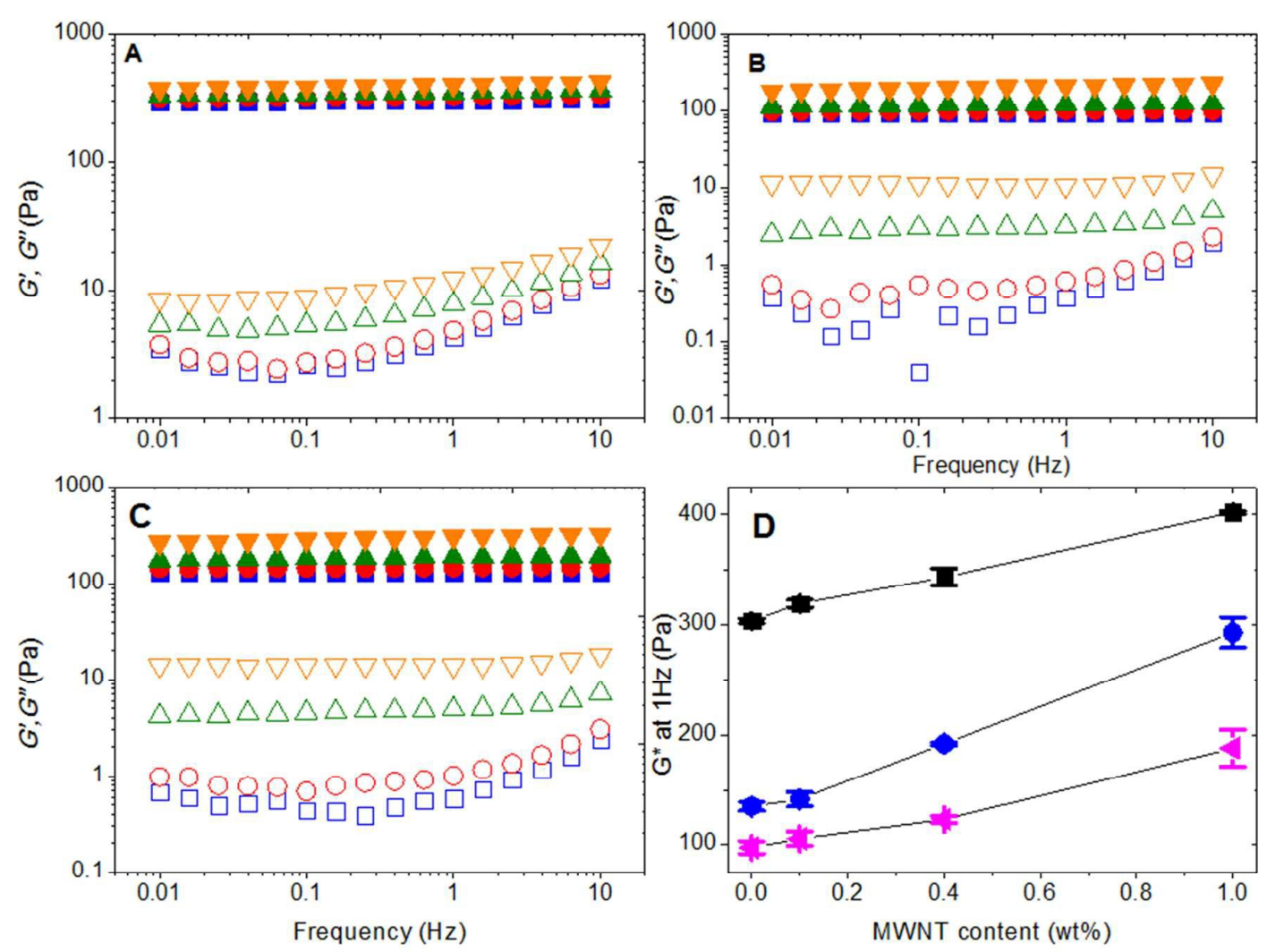
Fig. 2
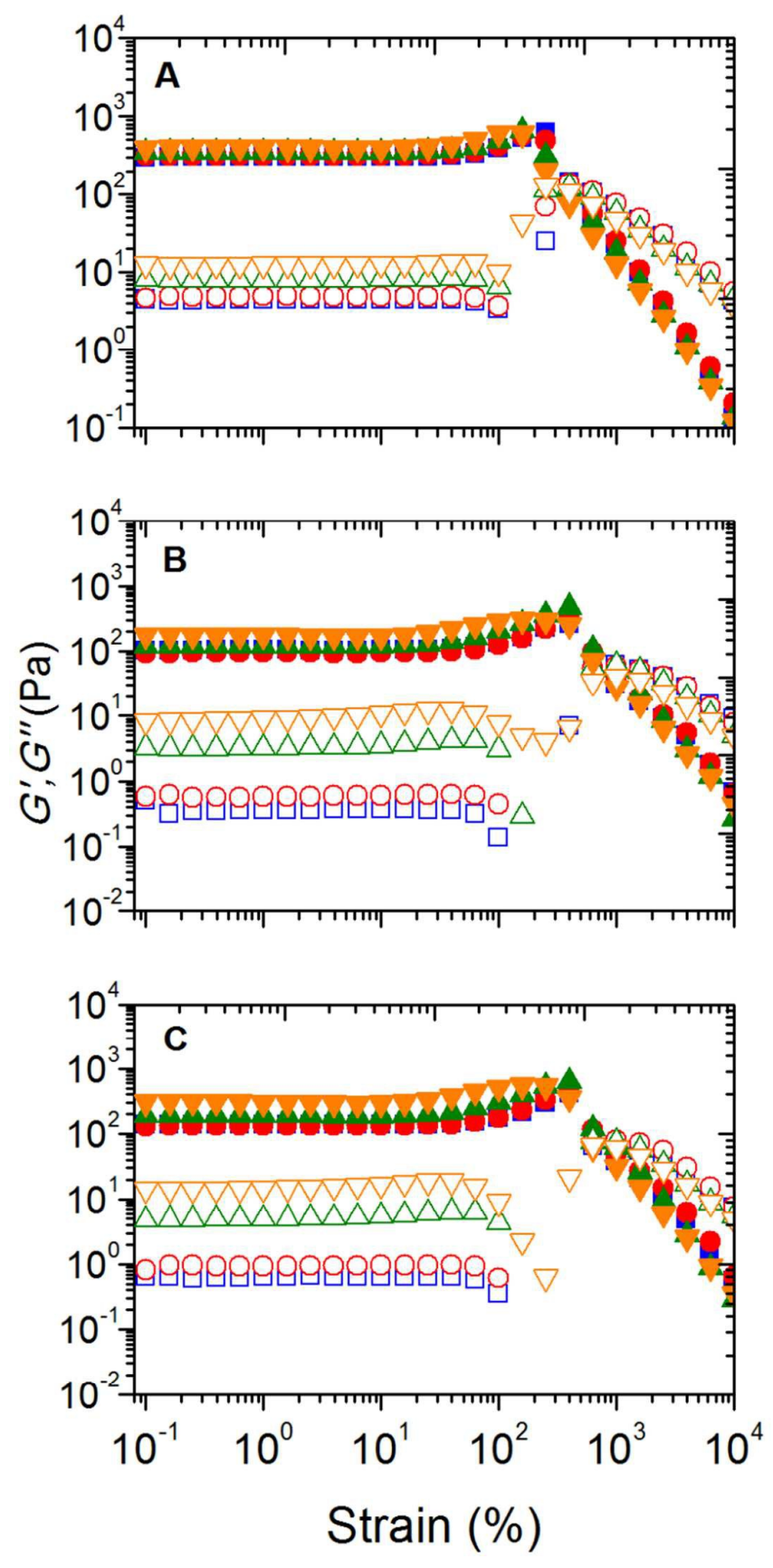
Fig. 3

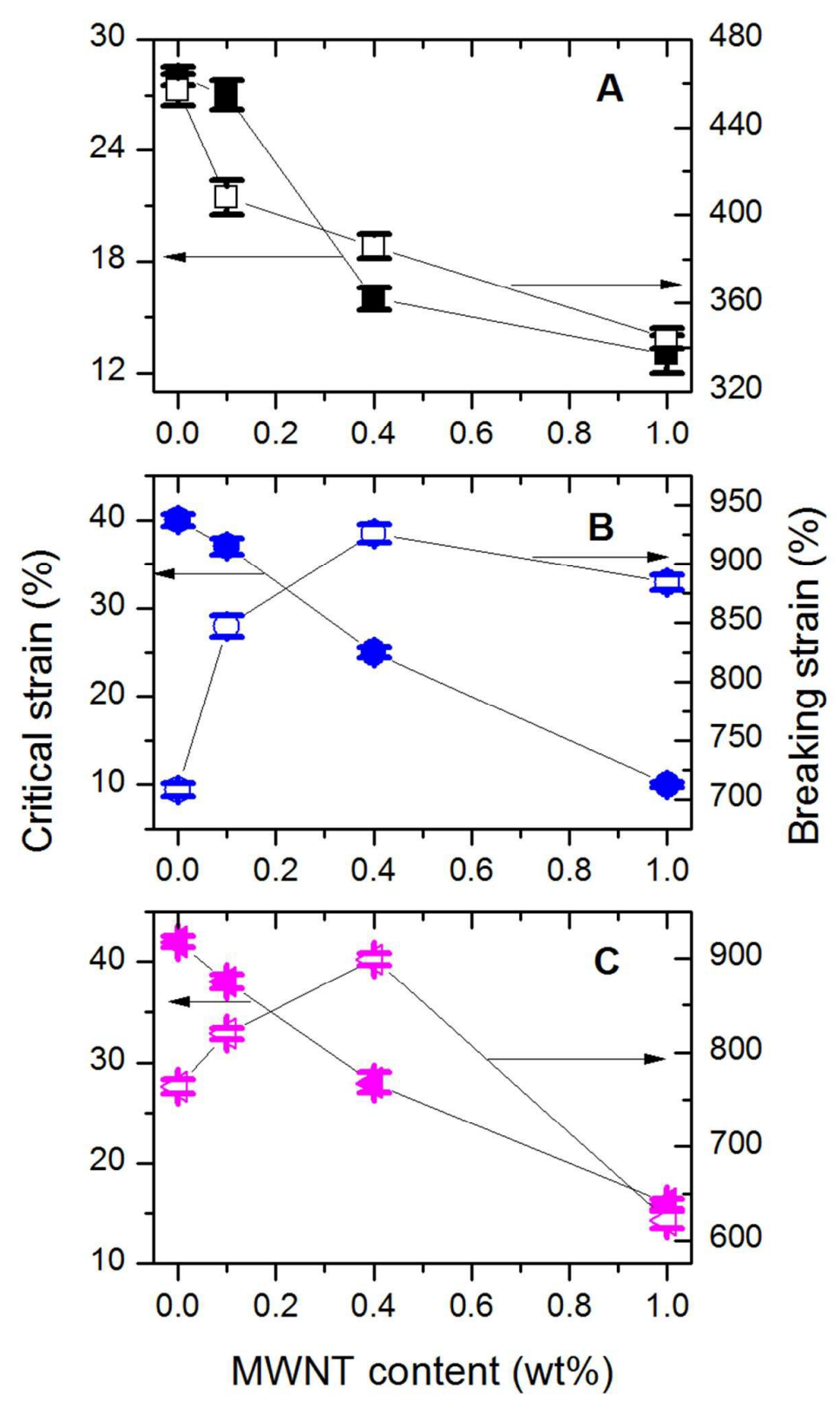


Fig. 4
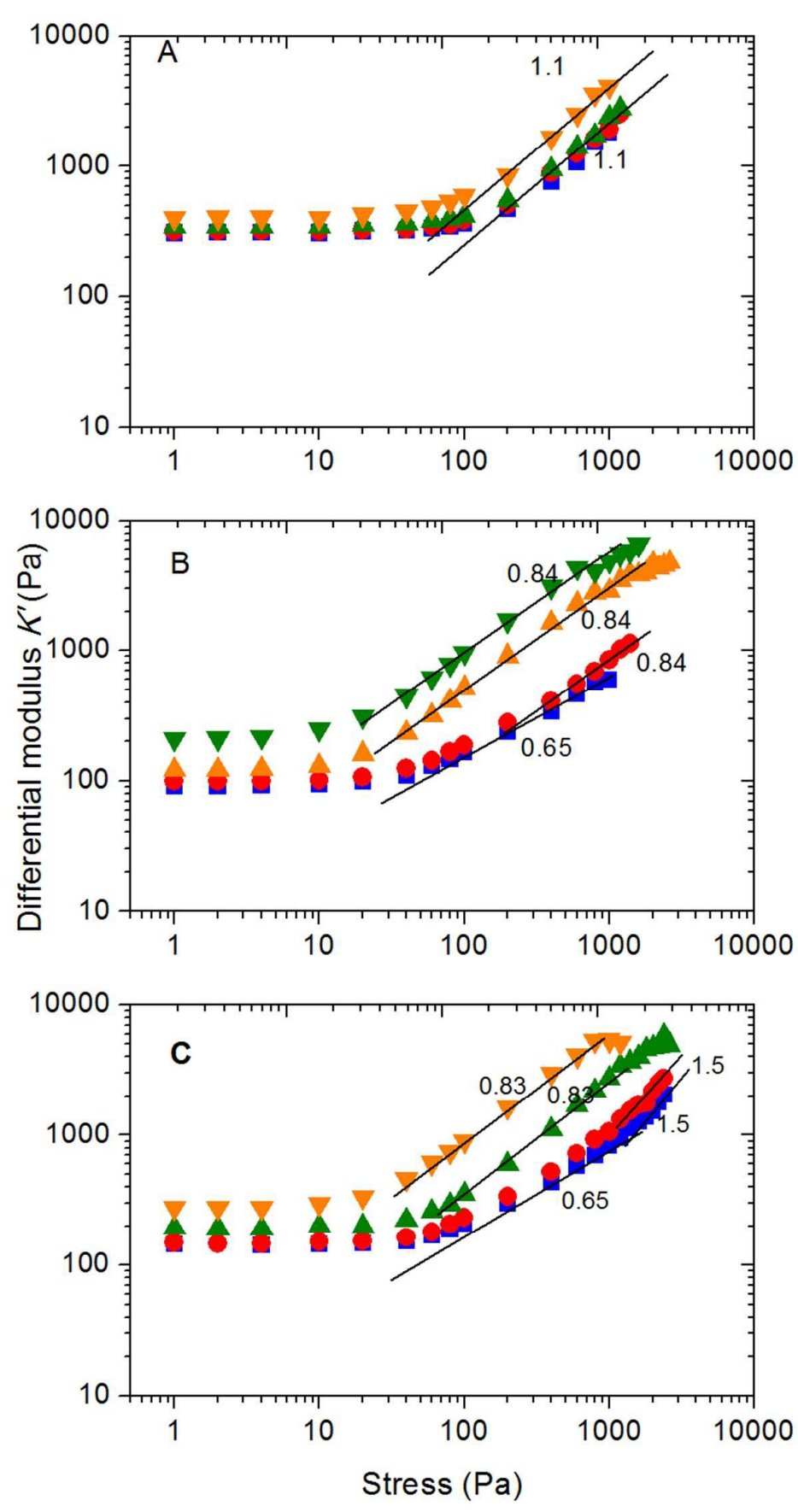
Fig. 5

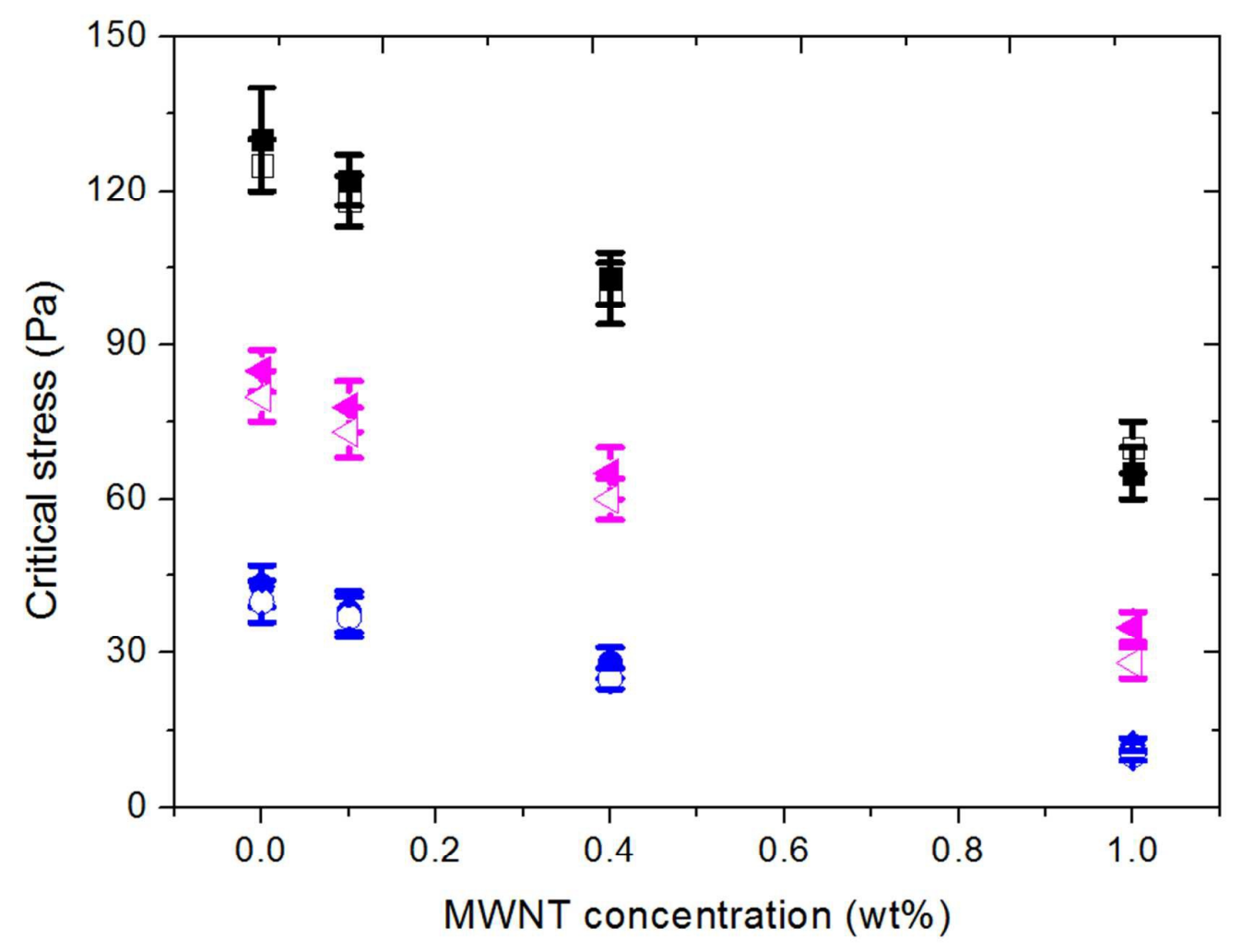


Fig. 6

A
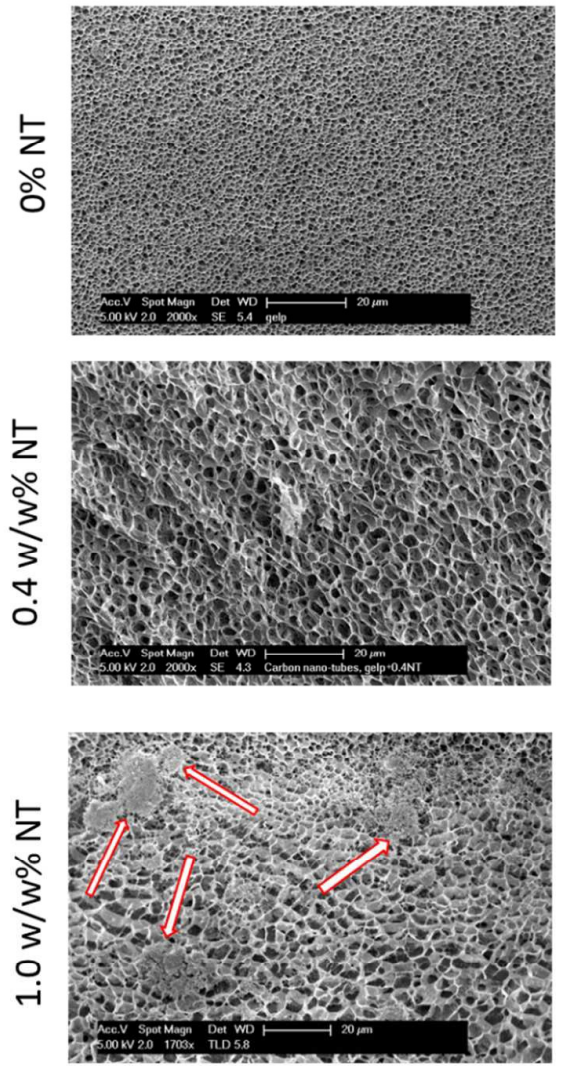

Physical gel
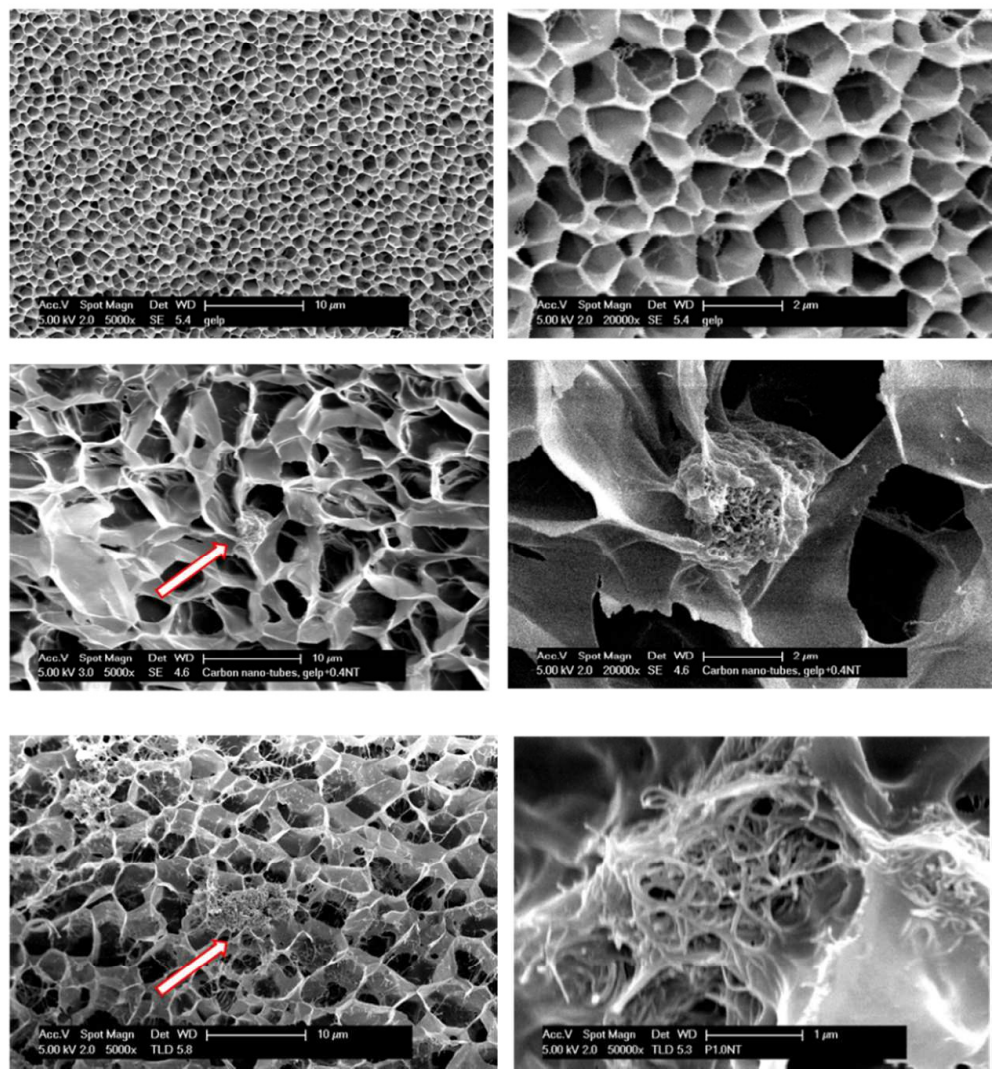

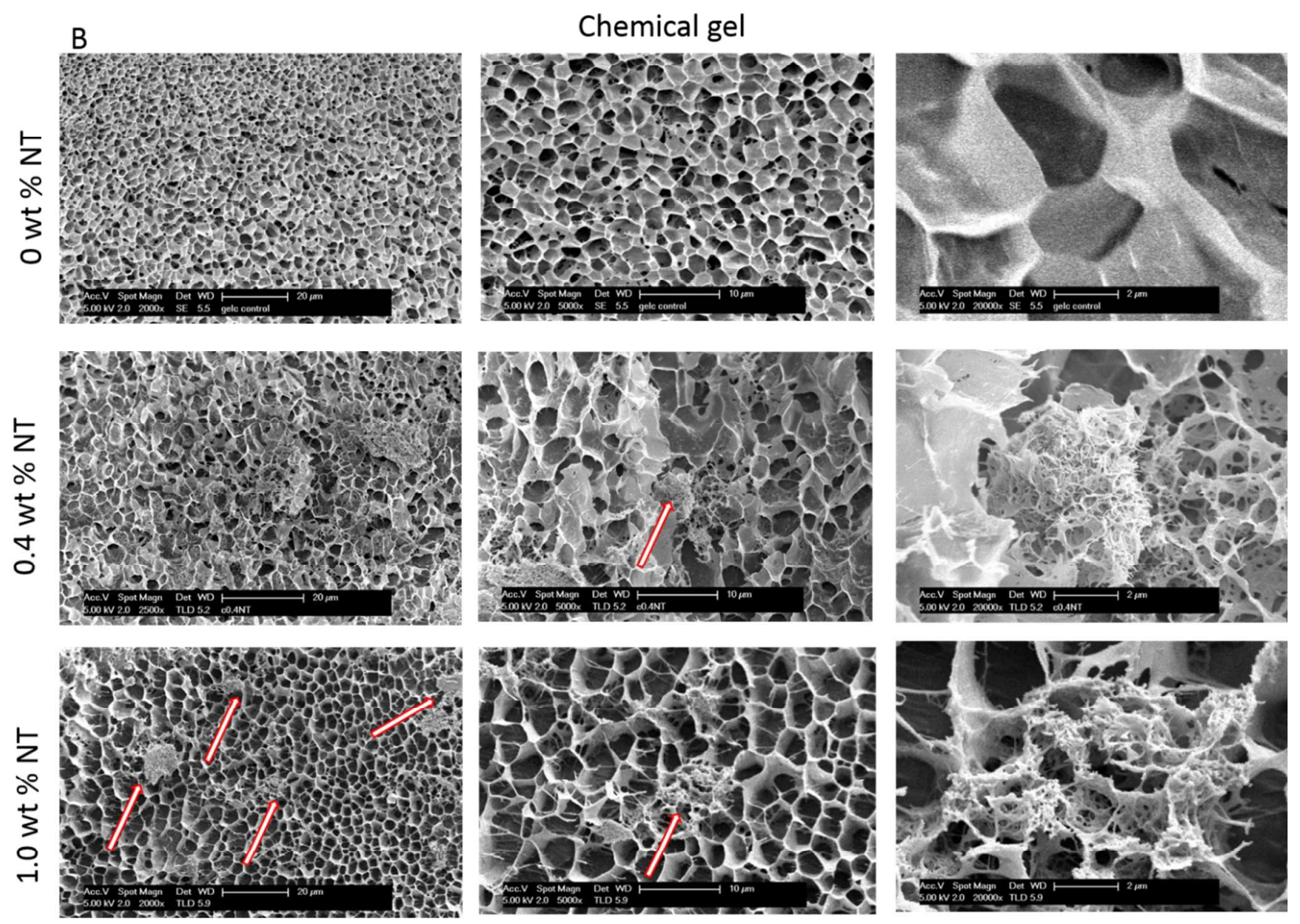

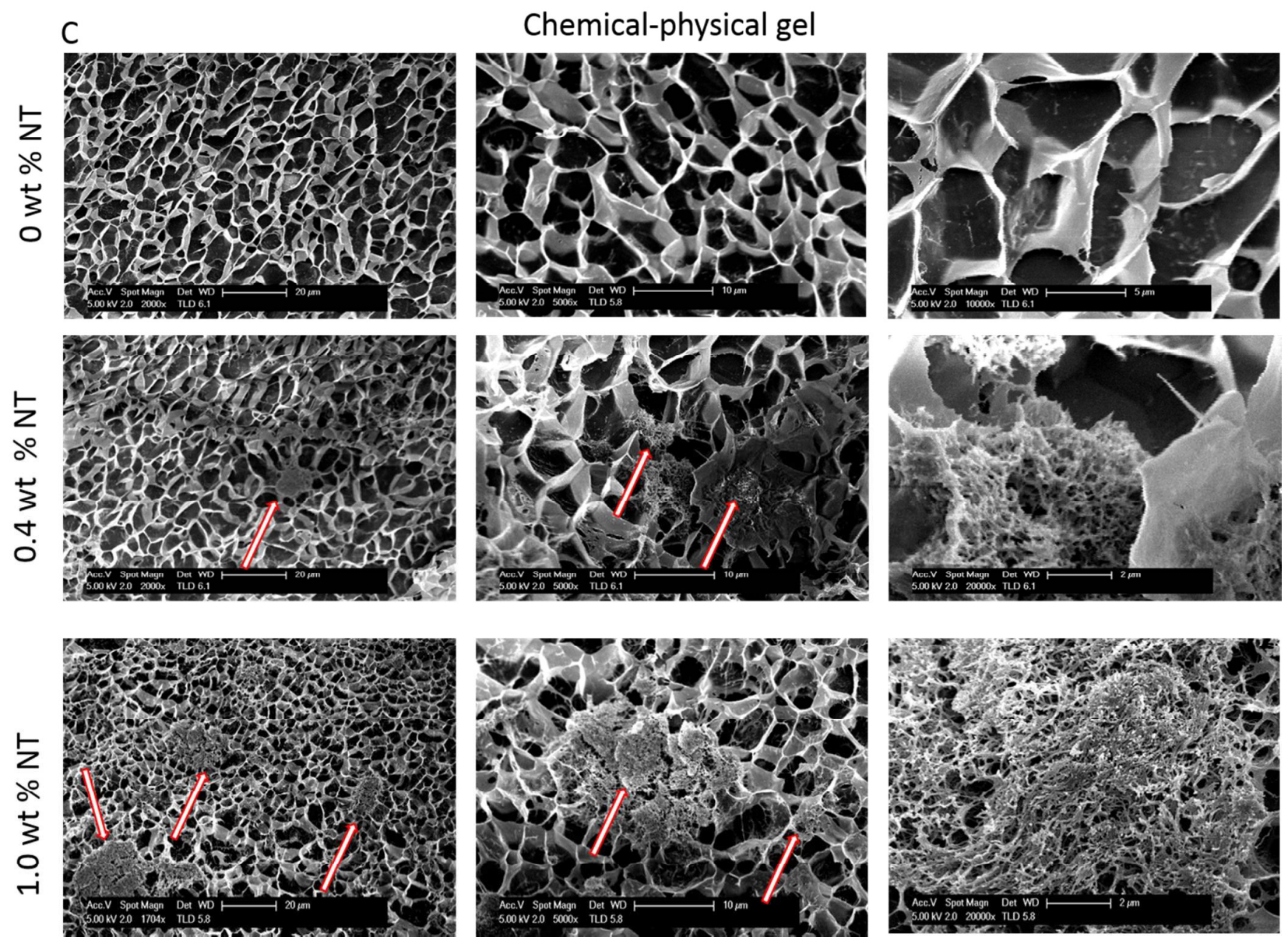
Fig.7

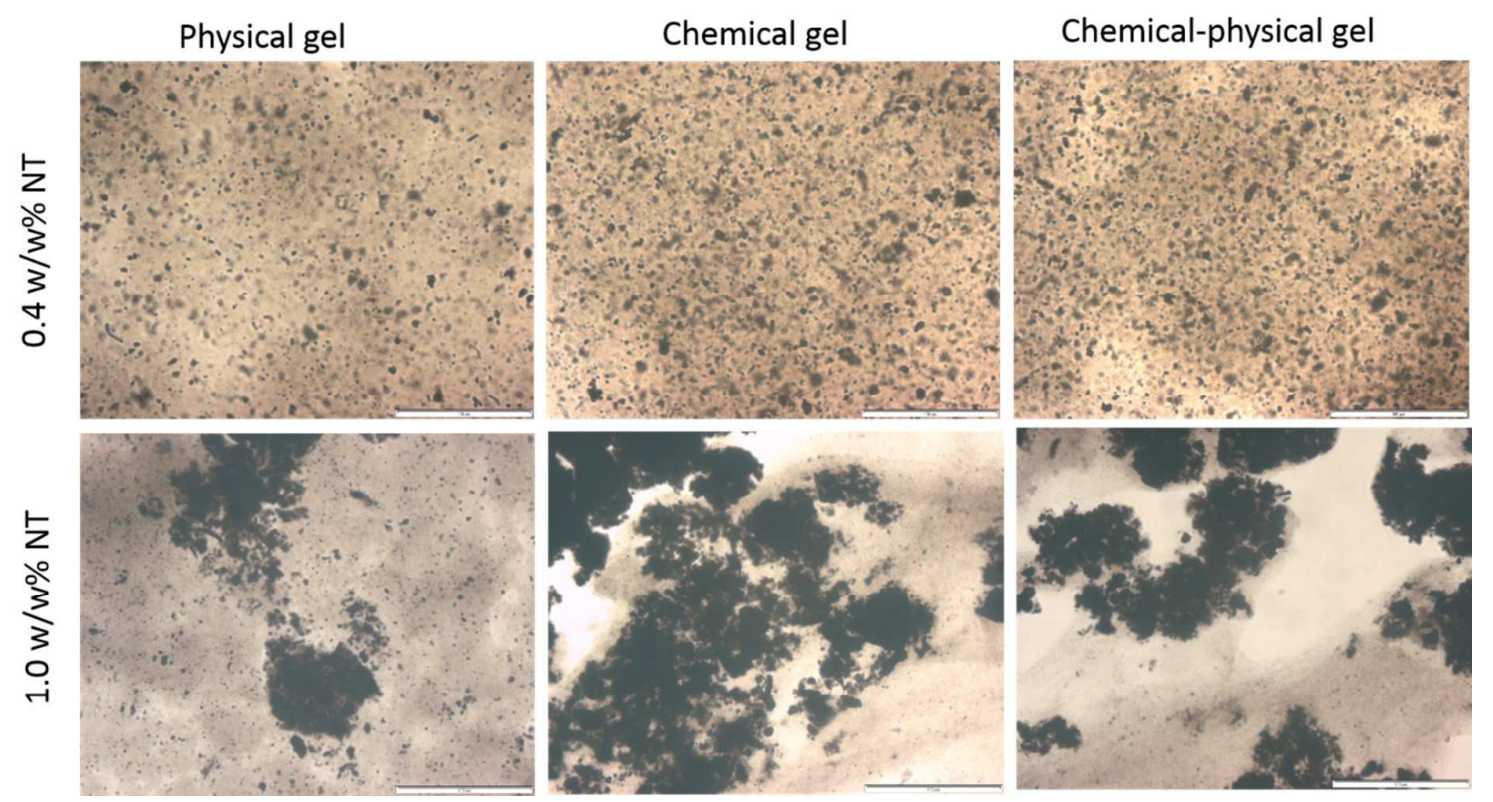


Fig.8

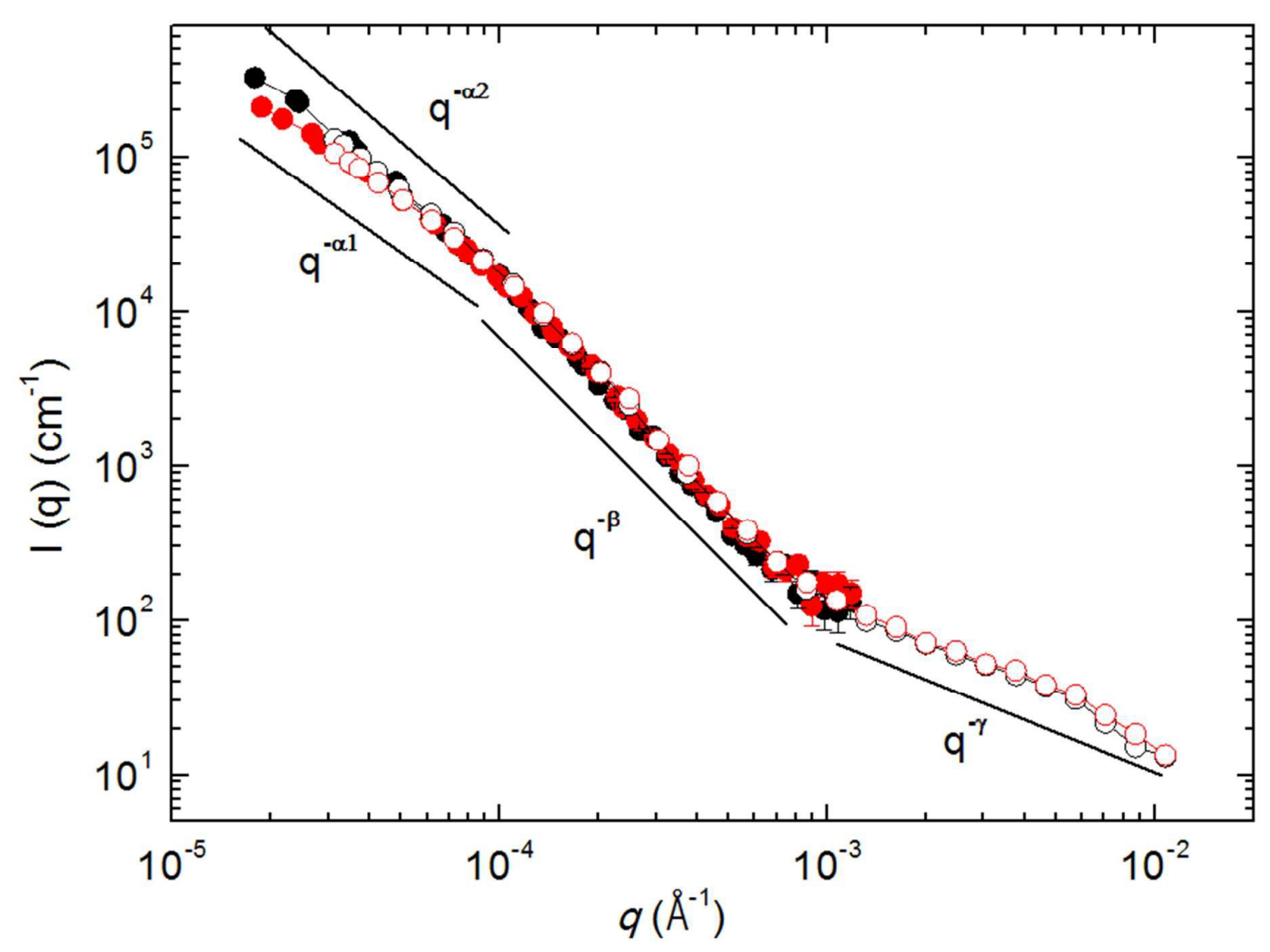

